BULLETIN OF THE

AMERICAN MATHEMATICAL SOCIETY

Volume 80, Number 1, January 1974

\title{
AN IMPLICIT FUNCTION THEOREM FOR SMALL DIVISOR PROBLEMS
}

\author{
BY E. ZEHNDER ${ }^{1}$
}

Communicated by S. S. Chern, June 18, 1973

1. Introduction. Various nonlinear problems, not accessible to standard existence theorems, led to new techniques which allowed the solution of the isometric embedding problem (J. Nash [1]) and stability problems of Hamiltonian systems connected with small divisors (A. N. Kolmogorov, V. I. Arnold, J. Moser [2]-[6]). Subsequently, the underlying ideas were abstracted as implicit function theorems [7]-[10], which however do not cover most small divisor problems. It is the aim of this paper to formulate and prove a simple implicit function theorem also covering many of these problems. The underlying idea is due to $\mathrm{H}$. Rüssmann [11]. Its basic idea is a modification of Newton's method in the framework of linear spaces and not in that of the group of coordinate transformations as it was used in [2]-[6], [14]. The proof of this theorem is elementary; the real difficulty, however, lies in showing that the assumptions can be met in the relevant applications. We mention as a new application the perturbation theory of invariant tori of dimension $m \leqq n$ of globally Hamiltonian diffeomorphisms defined on a $2 n$-dimensional symplectic manifold, in which we were able to verify those assumptions. The proof will be published elsewhere. I am indebted to J. Moser for acquainting me with small divisor problems.

2. Implicit function theorem. The following set up is prompted by $\mathrm{H}$. Jacobowitz [9] and L. Nirenberg [10]. We consider three one-parameter families of Banach spaces $X_{\sigma}, Y_{\sigma}, Z_{\sigma}$ in the closed unit interval: for $0 \leqq \sigma^{\prime} \leqq \sigma \leqq 1$,

$$
X_{0} \supseteq X_{\sigma^{\prime}} \supseteq X_{\sigma} \supseteq X_{1}
$$

(and analogous for $Y_{\sigma}$ and $Z_{\sigma}$ ) and with norms ||$_{\sigma}$ in $X_{\sigma}, \mid \mathbf{I}_{\sigma}$ in $Y_{\sigma}$ and $\mid l_{\sigma}$ in $Z_{\sigma}$ satisfying

$$
|f|_{\sigma^{\prime}} \leqq|f|_{\sigma}, \quad|u|_{\sigma^{\prime}} \leqq|u|_{\sigma}, \quad|z|_{\sigma^{\prime}} \leqq|z|_{\sigma}
$$

AMS (MOS) subject classifications (1970). Primary 35A35, 58C15, 70F15, 70H20.

${ }^{1}$ Research sponsored under AFOSR Grant 70-1866C. 
for $f \in X_{\sigma}, u \in Y_{\sigma}, z \in Z_{\sigma}$ and $0 \leqq \sigma^{\prime} \leqq \sigma$. In $Y_{\sigma}$ we have a second norm \|\|$_{\sigma}$ such that $\mid u \mathbf{|}_{\sigma} \leqq\|u\|_{\sigma}$ and $\|u\|_{\sigma^{\prime}} \leqq\|u\|_{\sigma}$ for $u \in Y_{\sigma}$ and $\sigma^{\prime} \leqq \sigma$. For fixed $N>0$ and $1 \geqq R>0$ we define the open balls

$$
N_{\sigma}=\left\{\left.f \in X_{\sigma}|| f\right|_{\sigma}<N\right\} \subset X_{\sigma}, \quad R_{\sigma}=\left\{\left.u \in Y_{\sigma}|| u\right|_{\sigma}<R\right\} \subset Y_{\sigma}
$$

and

$$
\hat{R}_{\sigma}=\left\{u \in Y_{\sigma} \mid\|u\|_{\sigma}<R\right\} \subset Y_{\sigma} .
$$

Let $F(\cdot, \cdot)$ be a mapping into $Z_{0}$ which is defined for every $(f, u) \in$ $X_{\sigma} \times Y_{\sigma}$ belonging to $N_{\sigma} \times R_{\sigma}$ for some $\sigma>0$, and which is continuous as a mapping from $N_{\sigma} \times R_{\sigma}$ into $Z_{\sigma^{\prime}}$ for every $0 \leqq \sigma^{\prime} \leqq \sigma$. The aim is to solve for a given $f$ in some $N_{\sigma}, F(f, u)=0, u$ in some $Y_{\sigma^{\prime}}, \sigma^{\prime}<\sigma$, assuming that $\left|F\left(f, u_{0}\right)\right|_{\sigma}$ is sufficiently small. We make the following hypothesis in which $\alpha, \beta, \gamma, K_{1}, K_{2}, K_{3}>0$ are fixed.

(i) Taylor formula. For every $\sigma$ in $0<\sigma \leqq 1$ and every $f \in N_{\sigma}$ the mapping $F(f, \cdot)$ from $R_{\sigma} \subset Y_{\sigma}$ into $Z_{\sigma^{\prime}}, \sigma^{\prime}<\sigma$, has a Fréchet derivative $d F(f, u)$ at every $u \in \hat{R}_{\sigma}$, and for $u, v \in \hat{R}_{\sigma}, Q(f ; u, v)=F(f, u)-F(f, v)-$ $d F(f, u)(u-v)$, satisfies:

$$
|Q(f ; u, v)|_{\sigma^{\prime}} \leqq\left(K_{1} /\left(\sigma-\sigma^{\prime}\right)^{\alpha}\right) \mathbf{|} u-v \mathbf{I}_{\sigma}^{2} .
$$

(ii) Approximative right inverse of $d F(f, u)$. For every $\sigma$ in $0<\sigma \leqq 1$ and every $(f, u) \in N_{\sigma} \times \hat{R}_{\sigma}$ there is a linear map $\eta(f, u)(\cdot) \in L\left(Z_{\sigma}, Y_{\sigma^{\prime}}\right)$ for every $\sigma^{\prime}<\sigma$, such that for all $a \in Z_{\sigma}$

$$
|(d F(f, u) \circ \eta(f, u)-\mathbf{1})(a)|_{\sigma^{\prime}} \leqq \frac{K_{2}}{\left(\sigma-\sigma^{\prime}\right)^{\alpha+\gamma}}|F(f, u)|_{\sigma} \cdot|a|_{\sigma}
$$

and

$$
\begin{aligned}
& \mathbf{|} \eta(f, u)(a) \mathbf{I}_{\sigma^{\prime}} \leqq\left(K_{3} /\left(\sigma-\sigma^{\prime}\right)^{\gamma}\right)|a|_{\sigma}, \\
& \|\eta(f, u)(a)\|_{\sigma^{\prime}} \leqq\left(K_{3} /\left(\sigma-\sigma^{\prime}\right)^{\gamma+\beta}\right)|a|_{\sigma} .
\end{aligned}
$$

THEOREM 1. Under the above conditions (i), (ii) there exist two constants $C_{1}$ and $C_{2}$ depending on $\alpha, \beta, \gamma, K_{1}, K_{2}$ and $K_{3}$ such that if $\left(f, u_{0}\right) \in N_{\sigma} \times \hat{R}_{\sigma}$ with $\left\|u_{0}\right\|_{\sigma} \leqq r<R$ for some $\sigma$ in $0<\sigma \leqq 1$ satisfies $\left|F\left(f, u_{0}\right)\right|_{\sigma} \leqq C_{1} \cdot(R-r) \cdot$ $\sigma^{q}, q \geqq 2 \gamma+\alpha+\beta$, then there exists a $u_{f} \in Y_{\sigma / 2}$ with $F\left(f, u_{f}\right)=0$. Further $u_{f}$ satisfies

$$
\mathbf{|} u_{f}-u_{0} \mathbf{I}_{o / 2} \leqq C_{2} \cdot\left|\left(F f, u_{0}\right)\right|_{\sigma} \cdot \sigma^{-\gamma}
$$

and

$$
\left\|u_{f}-u_{0}\right\|_{\sigma / 2} \leqq(R-r) \sigma^{\alpha-\gamma-\beta} .
$$

Proof. We use Newton's method but replace the inverse of $d F(f, u)$ (which need not exist) with the approximate right inverse $\eta(f, u)$ to define 
a sequence $\left(u_{n}\right), n \in \boldsymbol{Z}_{0}^{+}$which (as we prove) converges to a solution of $F(f, u)=0$. Let $K=\max \left\{1, K_{i}\right\}, 1 \leqq i \leqq 3, \lambda=4, \mu=\lambda / 2, \kappa=\frac{3}{2}, s=q^{-1}$ and $\exp (-\xi)=K^{-3} \cdot 2^{-3(q+1)}$. Set

$\sigma_{n}=\sigma / 2 \cdot\left(1+\exp \left(-\xi_{S \kappa^{n}}\right)\right), \quad \tau_{n+1}=\frac{1}{2}\left(\sigma_{n+1}+\sigma_{n}\right)$ for $n=0,1,2, \cdots$.

We then have $\sigma_{n+1}<\tau_{n+1}<\sigma_{n}$ and $\lim \sigma_{n}=\sigma / 2$ as $n \rightarrow \infty$. We define inductively the sequence $\left(u_{n}\right)$ by $u_{0}$ and

$$
u_{n+1}=u_{n}+v_{n}, \quad v_{n}=-\eta\left(f, u_{n}\right)\left(F\left(f, u_{n}\right)\right), \quad n \geqq 0 .
$$

To simplify the notation we omit the $f$ 's in the following. Using induction we prove the statements $S_{n}$ :

$$
\begin{aligned}
u_{n} \in Y_{\sigma n}, & \left|F\left(u_{n}\right)\right|_{\sigma_{n}} \leqq \nu(R-r) \cdot \sigma^{\alpha} \cdot \exp \left(-\xi \lambda \kappa^{n}\right), \\
v_{n} \in Y_{\tau n+1}, & \mid v_{n} \mathbf{I}_{\tau n+1} \leqq \nu(R-r) \cdot \sigma^{\alpha-\gamma} \exp \left(-\xi \mu \kappa^{n}\right), \\
& \left\|v_{n}\right\|_{\tau n+1} \leqq \nu(R-r) \cdot \sigma^{\alpha-\gamma-\beta} \exp \left(-\xi \mu \kappa^{n}\right),
\end{aligned}
$$

with $0<\nu \leqq 1$ to be determined later on. $S_{0}$ is valid if $\left|F\left(f, u_{0}\right)\right|_{\sigma} \leqq$ $v \cdot C_{1} \cdot(R-r) \cdot \sigma^{\alpha}$ with $C_{1}=\exp (-2 \lambda \xi)$. Assuming the validity of $S_{i}$, $i=1,2, \cdots, n$, we prove $S_{n+1}$. Since $u_{n+1}, u_{n} \in \hat{R}_{\tau_{n+1}} \subset \hat{R}_{s_{n+1}}$, it follows for

$$
F\left(u_{n+1}\right)=-\left(d F\left(u_{n}\right) \circ \eta\left(u_{n}\right)-\mathbf{1}\right)\left(F\left(u_{n}\right)\right)+Q\left(u_{n+1}, u_{n}\right)
$$

using (i) and (ii):

$$
\begin{aligned}
\left|F\left(u_{n+1}\right)\right|_{\sigma_{n+1}} & \leqq \frac{K_{2}}{\left(\sigma_{n}-\sigma_{n+1}\right)^{\alpha+\gamma}}\left|F\left(u_{n}\right)\right|_{\sigma_{n}}^{2}+\frac{K_{1}}{\left(\tau_{n+1}-\sigma_{n+1}\right)^{\alpha}}\left|\eta\left(u_{n}\right)\left(F\left(u_{n}\right)\right)\right|_{\tau_{n+1}}^{2} \\
& \leqq\left\{\frac{K_{2}}{\left(\sigma_{n}-\sigma_{n+1}\right)^{\alpha+\gamma}}+\frac{K_{1} K_{3}^{2}}{\left(\tau_{n+1}-\sigma_{n+1}\right)^{\alpha}\left(\sigma_{n}-\tau_{n+1}\right)^{2 \gamma}}\right\}\left|F\left(u_{n}\right)\right|_{\sigma_{n}}^{2} .
\end{aligned}
$$

From this estimate $(n+1) 1$ follows immediately. Using then (9) and (4), (5), (6) one easily verifies $(n+1)(2-4)$. From $(n 1)$ we now conclude that $F\left(f, u_{n}\right) \rightarrow 0$ in $Z_{\sigma / 2}$ as $n \rightarrow \infty$. Since $u_{n+1}-u_{n}=v_{n}$, it follows from ( $\left.n 2\right)$ that $\left(u_{n}\right)$ is a Cauchy sequence in $Y_{\sigma / 2}$. Calling $\lim u_{n}=u$ we conclude from the continuity of $F$, that $F(f, u)=0$. From (n3) we get for all $n$

$$
\begin{aligned}
\left\|u_{n}-u_{0}\right\|_{\sigma / 2} & \leqq \sum_{n=0}^{\infty}\left\|v_{n}\right\|_{\sigma / 2} \\
& \leqq 2 \sigma^{\alpha-\gamma-\beta}(R-r) \exp (-\xi \mu[\kappa-1])<(R-r) \sigma^{\alpha-\gamma-\beta}
\end{aligned}
$$


and hence (8). Similarly one gets (7); given $\left(f, u_{0}\right)$ such that

$$
0<\left|F\left(f, u_{0}\right)\right|_{\sigma} \leqq C_{1} \cdot(R-r) \sigma^{q}
$$

one chooses $v$ such that $\left|F\left(f, u_{0}\right)\right|_{\sigma}=v \cdot C_{1} \cdot(R-r) \sigma^{\alpha}$.

The new idea consists of introducing an approximate right inverse $\eta(f, u)$, see (4), which is an exact right inverse at the solutions of $F(f, u)=$ 0 ! For many small divisor problems such an approximate right inverse can be provided if one is dealing with a conjugation problem.

REMARK (SEE [15]). Various modifications of Theorem 1 are possible. Under additional conditions for $F$ and $\eta$ the above solution $f \rightarrow u_{f}$ of $F\left(f, u_{f}\right)=0$ is Fréchet differentiable even though the problem may not have a unique solution. Uniqueness of the solutions $u_{f}$ follows from the existence of an approximative left inverse. A similar theorem holds in the framework of spaces of differentiable functions using smoothing operators.

3. An application. For motivation and background consult J. Moser [12] and S. Graff [13]. We consider on the manifold $M=T^{n} \times \boldsymbol{R}^{n} \times \boldsymbol{R}^{m} \times \boldsymbol{R}^{m}$ the real analytic mapping

$$
\varphi_{0}:(x, y, \xi, \eta) \rightarrow\left(x+\omega+y, y, \Lambda^{+}(x) \xi, \Lambda^{-}(x) \eta\right),
$$

where $\Lambda^{ \pm}(x) \in L^{-1}\left(\boldsymbol{R}^{m}\right)$ with $\sup _{x \in T^{n}}\left(\left\|\Lambda^{+}(x)^{-1}\right\|,\left\|\Lambda^{-}(x)\right\|\right) \leqq a<1$, and where $\omega=\left(\omega_{1}, \cdots, \omega_{n}\right)$ satisfies

$$
|(\omega, k)-l| \geqq \gamma|k|^{-\beta},
$$

for all integers $(k, l), k=\left(k_{1}, \cdots, k_{n}\right) \neq 0,0<\gamma<\frac{1}{2}, \beta>n$. The question is, when does the invariant torus $T_{0}=T^{n} \times(\zeta=0), \zeta=(y, \xi, \eta)$ survive under a perturbation by a real analytic mapping $f$ defined in an open neighborhood of $T_{0}$. With $\left(\varphi_{\lambda}\right)$ we denote the family of mappings

$$
\varphi_{\lambda}:(x, y, \xi, \eta) \rightarrow\left(x+\omega+y, y,\left(\Lambda^{+}(x)+\lambda^{+}(x)\right) \xi,\left(\Lambda^{-}(x)+\lambda^{-}(x)\right) \eta\right),
$$

where $\lambda$ stands for the pair $\left(\lambda^{+}, \lambda^{-}\right)$. For a given mapping $f=\left(f_{i}\right), 1 \leqq i \leqq 4$, on $M$ and $\mu=\left(\mu_{0}, \mu_{1}\right) \in \boldsymbol{R}^{n} \times L\left(\boldsymbol{R}^{n}\right)$ we define $f_{\mu}=\left(f_{1}, f_{2}+\mu_{0}+\mu_{1} y, f_{3}, f_{4}\right)$. With

$$
\Omega_{\sigma \rho}=\Omega_{\sigma} \times \Omega_{\rho}=\{x|| \operatorname{Im} x \mid<\sigma\} \times\{|\zeta|<\rho\}
$$

we denote complex neighborhoods of the torus $T_{0}$.

THEOREM 2. Given $\varphi_{0}$ as above, then for every $\varepsilon>0$ there exists a $\delta>0$, $\delta\left(\varepsilon, \Omega_{\sigma \rho}, \varphi_{0}\right)$, such that for every real analytic mapping $f$ with $\left|f-\varphi_{0}\right|_{\Omega_{\sigma \rho}}<\delta$ there exist two real analytic mappings $\varphi_{\lambda}$ and $\psi: \Omega_{\sigma / 2 \rho} \rightarrow \Omega_{\sigma \rho}, \psi=\mathrm{id}+w$, with $w(x, \zeta)=\alpha(x)+(\beta(x), \zeta)$, and there exist constants $\mu \in\left(\boldsymbol{R}^{n}, L\left(\boldsymbol{R}^{n}\right)\right)$ such that

$$
\max \left\{|\mu|,|\alpha|_{\Omega_{\sigma / 2}},|\beta|_{\Omega_{\sigma / 2}},\left|\lambda^{ \pm}\right|_{\Omega_{\sigma / 2}}\right\}<\varepsilon
$$


and

$$
\left.T \psi \circ T \varphi_{\lambda}\right|_{T_{0}}=\left.T f_{\mu} \circ T \psi\right|_{T_{0}},
$$

where $T_{0}$ denotes the complex torus $\Omega_{\sigma / 2} \times\{0\}$ and $T$ the tangent functor.

We look at the mappings $f_{\mu} \circ(\mathrm{id}+w)-(\mathrm{id}+w) \circ \varphi_{\lambda}=\phi(f, u)$ on $\Omega_{\sigma \rho}$, where (according to the notation in Theorem 2) $u=(\mu, \lambda, \alpha, \beta)$ is an element of the Banach space $Y_{\sigma}$ of vector- and matrix-valued real analytic functions defined on the complex torus $\Omega_{\sigma}$. One then proves that the functional $F$, defined by $F(f, u)=\left(\left.\phi(f, u)\right|_{\zeta=0},\left.d(\phi(f, u))\right|_{\zeta=0}\right), d$ the Jacobian, satisfies the assumption of Theorem 1 . If $f$ and $\varphi_{0}$ are globally Hamiltonian mappings (i.e. $f^{*} \theta-\theta=d s$, with $\theta=\sum_{i=1}^{n} y_{i} d x_{i}+\sum_{j=1}^{m} \eta_{j} d \xi_{j}$ and $s$ a function defined on an open neighborhood of $T_{0}$ ) then one can show that $\mu=0$ in Theorem 2 .

COROLLARY. Let $f$ and $\varphi_{0}$ be real analytic globally Hamiltonian mappings such that $\left|f-\varphi_{0}\right|_{\Omega_{\sigma} \rho}$ is sufficiently small, then there exist two mappings $\psi$ and $\varphi_{\lambda}: \Omega_{\sigma / 2 \rho} \rightarrow \Omega_{\sigma \rho}$ such that

$$
\left.T \psi \circ T \varphi_{\lambda}\right|_{T_{0}}=\left.T f \circ T \psi\right|_{T_{0}},
$$

$T$ and $T_{0}$ as in Theorem 2. Moreover the local stable and unstable manifolds of the (under $f$ ) invariant torus $\psi\left(T_{0}\right)$ are real analytic and Lagrangian.

Using the methods of J. Moser [6], [9] we get a perturbation theory for hyperbolic tori in the differentiable case. The proofs will appear elsewhere.

\section{REFERENCES}

1. J. Nash, The imbedding problem for Riemannian manifolds, Ann. of Math. (2) 63 (1956), 20-63. MR 17, 782.

2. A. N. Kolmogorov, Théorie générale des systèmes dynamiques et mécanique classique, Proc. Internat. Congress Math. Amsterdam, vol. I, North-Holland, Amsterdam, 1957, pp. 315-333. MR 20 \#4066.

3. V. I. Arnol'd, Small denominators and problems of stability of motion in classical and celestial mechanics, Uspehi Mat. Nauk 18 (1963), no. 6 (114), 91-192=Russian Math. Surveys 18 (1963), no. 6, 85-191. MR 30 \#943.

4. J. Moser, On invariant curves of area-preserving mappings of an annulus, Nachr. Akad. Wiss. Göttingen Math.-Phys. Kl. II, 1962, 1-20. MR 26 \#5255.

5. - A new technique for the construction of solutions of nonlinear differential equations, Proc. Nat. Acad. Sci. U.S.A. 47 (1961), 1824-1831. MR 24 \#A2695.

6. - A rapidly convergent iteration method and non-linear partial differential equations, Ann. Scuola Norm. Sup. Pisa (3) 20 (1966), I: pp, 265-315, II: pp. 499-535. MR 33 \#7667; 34 \#6280.

7. J. T. Schwartz, Nonlinear functional analysis, Lecture notes, 1964, Gordon and Breach, New York, 1969, pp. 33-44.

8. F. Sergeraert, Un théorème de fonctions implicites sur certains espaces de Fréchet et quelques applications, Ann. Sci. École Norm. Sup., $4^{\mathrm{e}}$ série, 5 (1972), 599-660. 
9. H. Jacobowitz, Implicit function theorems and isometric embeddings, Ann. of Math. (2) 95 (1972), 191-225.

10. L. Nirenberg, An abstract form of the nonlinear Cauchy-Kowalewski theorem, J. Differential Geometry 6 (1972), 561-576.

11. H. Rüssmann, Kleine Nenner. II: Bemerkungen zur Newtonschen Methode, Nachr. Akad. Wiss. Göttingen Math.-Phys. Kl. (1972), 1-10.

12. J. Moser, Stable and random motions in dynamical systems with special emphasis on celestial mechanics, Princeton Univ. Press, Princeton, N.J., 1973.

13. S. Graff, On the conservation of hyperbolic invariant tori for Hamiltonian systems, Dissertation, New York University, New York, June, 1971.

14. S. Sternberg, Celestial mechanics, Part II, Benjamin, New York, 1969.

15. E. Zehnder, The Moser-Nash implicit function theorem for small divisor problems (to appear).

School of Natural Sciences, Institute for Advanced Study, Princeton, New JERSEY 08540 